\title{
Método ultrassônico de detecção de microvibração: Desenvolvimento da técnica
}

\author{
José Francisco Silva Costa Júnior*, João Carlos Machado
}

Resumo Nos últimos anos muitos pesquisadores têm-se dedicado à elaboração de um método capaz de gerar a propagação de onda de cisalhamento em um meio biológico e extrair informações referentes às microvibrações do meio causadas pela propagação dessa onda, o que permite caracterizar os parâmetros viscoelásticos do meio. Diante do potencial em se trabalhar com onda de cisalhamento, este trabalho apresenta um método ultrassônico de detecção de microvibração (DUmV), o qual faz uso do filtro de Kalman para estimar a vibração do meio frente a um sinal contaminado de ruído. O DUmV foi testado empregado-se um alto-falante para gerar microvibrações em uma membrana e um sistema ultrassônico operando em 4,96 MHz e no modo pulso-eco, para detectar as vibrações mencionadas. O DUmV se mostrou bastante promissor, pois conseguiu estimar vibrações quanto à frequência e às amplitudes da mesma ordem de grandeza que os valores encontrados na literatura (cerca de $10 \mu \mathrm{m}$ ). Além disso, essa técnica também mostrou a relação na qual o aumento da frequência de vibração implicou na redução da amplitude do sinal estimado. A metodologia desenvolvida no presente trabalho poderá ser empregada para se estimar a velocidade de fase e o coeficiente de atenuação de uma onda de cisalhamento propagando-se em um meio viscoelástico.

Palavras-chave Ultrassom, Onda de cisalhamento, Microvibrações, Filtro de Kalman.

\section{Ultrasonic method of microvibration detection: Development of the method}

\begin{abstract}
Many investigations have been devoted, during the last years, to implement an ultrasonic method able to generate shear waves and to detect the microvibrations caused by the wave propagation in biological tissues. Applications of such method include measuring rheological parameters for tissue characterization. Considering the potential to use shear wave propagation, this work presents an ultrasonic method able to detect microvibrations (UDmV), which is based on Kalman filtering to estimate the medium vibrations from a signal contaminated with noise. The UDmV was tested employing a speaker to generate microvibrations on a membrane and a pulse-echo ultrasonic system, operating at a center frequency of $4.96 \mathrm{MHz}$, to detect the membrane microvibrations. The UDmV demonstrated promising future potential, being able to detect the vibration frequency and amplitude with the same order of magnitude as presented in the literature $(\approx 10 \mu \mathrm{m})$. Moreover, the method implemented in the present work confirmed previous results, from the literature, related with a reduction of the estimated vibration amplitude as the vibration frequency increases. The method developed in the present work can be employed to estimate the phase velocity and the attenuation coefficient of a shear wave propagating in a viscoelastic medium.
\end{abstract}

Keywords Ultrasound, Shear wave, Microvibrations, Kalman filter. 


\section{Extended Abstract}

\section{Introduction}

Measurement of rheological parameters based on microvibrations caused by shear wave propagation is an important tool for tissue characterization. This work presents an ultrasonic method able to detect microvibrations (UDmV), which is based on Kalman filtering to estimate the medium vibrations from a signal contaminated with noise. The UDmV was tested employing a speaker to move a membrane with microvibrations and a pulse-echo ultrasonic system, operating at a center frequency of $4.96 \mathrm{MHz}$, to detect the membrane microvibrations.

\section{Method}

An experimental setup (Figure 1) was used to detect polyurethane membrane vibrations imposed by an attached speaker with maximum power of $3 \mathrm{~W}$. The membrane vibrations were detected using an ultrasonic system, containing a TB 1000 board configured to operate in a pulse-echo mode and a ultrasonic transducer, $T_{\text {det }}$ with center frequency of $4.96 \mathrm{MHz}$. The TB 1000 board is a specially designed computer board, installed in the ISA bus, that functions as a transmit/receive tone burst ultrasonic system. The ultrasonic transducer was driven by a sinusoidal burst and the emitted ultrasonic beam was perpendicularly incident to the membrane surface.

The echo signals from the membrane were captured with a digital oscilloscope and transferred to a microcomputer, by an USB interface, where they were processed including: segmentation of the burst signals, alignment of all segments and demodulation of the radiofrequency echoes using in phase and quadrature detection. The demodulated components of the echo signals were employed to determine its phase, corresponding to the middle of the echo envelope, which is proportional to membrane vibration waveform. The membrane vibration waveform was determined using Kalman filtering over the vibration signal detected with the ultrasonic system, which was contaminated with noise. This filter is a linear estimator, which uses a state space model to estimate, recursively, the state variables according to the minimum mean square error (MMSE).

\section{Results}

The method was tested with different conditions regarding the membrane vibrations, with estimated amplitude ranging from 1.33 to $20.24 \mathrm{~mm}$ and at frequencies of 150 and $200 \mathrm{~Hz}$. As the vibration frequency must be known to run the Kalman filter (Zheng et al., 2007), it was necessary to span the frequency values (from 10 to $750 \mathrm{~Hz}$, in steps of $1 \mathrm{~Hz}$ ) used in the filter to find out the estimated frequency based on the maximum filter output vibration amplitude. The estimated frequency was compared with the one from the function generator used to feed the loudspeaker. Then next step in the experiment was to use the Kalman filter to detect the membrane vibration frequency, while the speaker was excited with a sinusoidal signal with 12 Veak-to-peak amplitude. As the frequencies used in the function generator were 100, 130,150,180, 200 and $280 \mathrm{~Hz}$, the corresponding estimated frequencies were 99, 128, 149, 178, 197 and $272 \mathrm{~Hz}$, respectively.

\section{Discussion and Conclusion}

Considering the results obtained in the present work, the method can be used to estimate the shear wave phase speed and attenuation coefficient, which, in turn, can be used to characterize biological media through the coefficients of shear elasticity and viscosity.

Although the UDmV system was not validated to measure amplitude vibrations, the detected amplitude vibrations are on the same order of magnitude as presented in the literature $(\approx 10 \mu \mathrm{m})$. Regarding the vibration frequency, the method estimated it with an accuracy of better than $3 \%$ 


\section{Introdução}

Ultimamente tem havido um esforço da comunidade científica para usar ondas de cisalhamento para a caracterização da viscoelasticidade de meios biológicos. A motivação pelo emprego de ondas de cisalhamento deve-se a grande variabilidade (cerca de 7 ordens de grandeza) do módulo de cisalhamento entre os diferentes meios biológicos, ao passo que o módulo volumétrico varia apenas de uma ordem de grandeza. Com isto, a propagação das ondas de cisalhamento torna mais viável a caracterização de meios biológicos através de parâmetros viscoelásticos (Sarvazyan et al., 1998). Uma técnica bastante utilizada para examinar alterações nas propriedades mecânicas de um meio, como por exemplo, o coeficiente de elasticidade de cisalhamento do tecido mole, é a elastografia, a qual pode estar baseada na propagação de ondas de cisalhamento no meio (Benech, 2004; Bercoff et al., 2003; 2004; Chen et al., 2009; Deffieux et al., 2009; Gennisson et al., 2004; Gennisson e Cloutier, 2006; Giannoula e Cobbold, 2007; 2008; 2009; Nightingale et al., 2001; 2002; 2003; Rengaraju et al., 2009; Sarvazyan et al., 1998; Urban et al., 2009).

Uma das motivações para isso reside no fato de ser consolidado, em medicina clínica, que propriedades mecânicas tais como a dureza ou módulo elástico são normalmente diferentes entre tecidos biológicos sadios e com anomalias, assim como entre os tecidos na vizinhança de alguma anomalia com aqueles que formam a própria anomalia (Anderson, 1985; Carstensen et al., 2008; Greenleaf et al., 2003; Kruse et al., 2000). Ao longo de aproximadamente 20 anos, a elastografia revelou-se um método de grande potencial para detectar anomalias no tecido biológico, por isso atualmente há na literatura vários trabalhos aplicando elastografia principalmente às detecções de câncer de mama (Garra et al., 1997; Melodelima et al., 2006; 2007; Zhu et al., 2008), câncer de próstata (Cochlin et al., 2002; Kamoi et al., 2008), coagulação sanguínea (Gennisson et al., 2004; 2006), fibrose hepática (Castera et al., 2008; De Lédinghen e Vergniol, 2008; Nitta et al., 2002; Sporea et al., 2008) e doenças pancreáticas (Saftoiu e Vilman, 2006).

Alguns pesquisadores propuseram configurações experimentais relacionadas à elastografia, nas quais foram empregadas uma fonte de vibração mecânica externa com baixa frequência, para induzir a propagação da onda transversal no meio, e um sistema de ultrassom pulso-eco (Benech, 2004; Catheline et al., 1999a; 1999b; 2000; 2004; Gennisson et al., 2004; Gennisson e Cloutier, 2006) ou o Doppler ultrassônico (Gao et al., 1993; 1995; Yamakoshi et al., 1990), para detectar a vibração do meio imposta pela propagação da onda de cisalhamento, a qual induz, tipicamente, vibrações da ordem de $20 \mu \mathrm{m}$ em meios biológicos (Bercoff et al., 2004; Chen et al., 2009; Zheng et al., 2007).

Com o mesmo intuito, foi desenvolvido no presente trabalho um método utrassônico de detecção de microvibração (DUmV) do meio, o qual poderá ser utilizado na determinação da propagação de uma onda de cisalhamento em um tubo ou em um phantom de gelatina através da medição da velocidade de fase e do coeficiente de atenuação.

\section{Materiais e Métodos}

\section{Modelagem matemática}

Segundo Zheng et al. (2003), o ponto de partida consiste em equacionar a vibração do meio, $d(t)$ como:

$d(t)=D \cdot \operatorname{sen}\left(\omega_{s} t+\varphi_{s}\right)$

onde $D$ e $\varphi_{s}$ representam a amplitude e a fase da vibração, respectivamente. A derivada temporal de $d(t)$ resulta na expressão para a velocidade $u(t)$ causada pela vibração e dada por:

$u(t)=D \omega_{s} \cdot \cos \left(\omega_{s} t+\varphi_{s}\right)$

Considerando que o sistema de ultrassom empregado para detectar a vibração do meio funciona da forma pulso-eco, emitindo uma salva de senoides com frequência $\omega_{0}$ e período de repetição de pulsos (PRP) $T$, o $n$-ésimo sinal de eco ultrassônico captado pelo transdutor é representado por (Zheng et al., 2007):

$r(t, n)=\operatorname{Re}\left[g(t, n) e^{i\left(\omega_{0} t+\varphi_{0}\right)}\right]$

Onde $g(t, n)$ é o envelope complexo de $r(t, n), \varphi_{0}$ é uma fase inicial e $\operatorname{Re}[x]$ representa a parte real de $x$. O envelope complexo pode ser escrito em função de uma fase $\phi(t, n)$ e da amplitude $|g(t, n)|$, ou dos componentes em fase $I(t, n)$, e quadratura $Q(t, n)$, como pode ser observado a seguir (Zheng et al., 2007):

$$
g(t, n)=I(t, n)+i Q(t, n)=|g(t, n)| e^{i \varphi(t, n)}
$$

Uma forma de extrair o sinal de representação da vibração do meio consiste em aplicar, inicialmente, um demodulador de fase e quadratura em (3), o que resulta nas equações dos componentes em fase $I$, e quadratura $Q$, (Zheng et al., 2003; 2004; 2007):

$I(t, n)=0,5 \cdot|g(t, n)| \cdot \cos \left\{\beta \operatorname{sen}\left[\omega_{\mathrm{s}}(t+n T)+\varphi_{s}\right]+\varphi_{0}\right\}$ 
$Q(t, n)=-0,5 \cdot|g(t, n)| \cdot \operatorname{sen}\left\{\beta \operatorname{sen}\left[\omega_{\mathrm{s}}(t+n T)+\varphi_{s}\right]+\varphi_{0}\right\}(6)$

onde $\beta=2 D \omega_{0} \cos (\theta) / c, c$ é a velocidade de propagação da onda longitudinal no meio estudado e $\theta$ é o ângulo entre o feixe ultrassônico e a direção de vibração deste meio.

A representação do sinal de vibração do meio $s(t, n)$ é dada por:

$$
s(t, n)=-\frac{1}{2 \operatorname{sen}\left(\omega_{\mathrm{s}} T / 2\right)} \tan ^{-1}\left[\frac{Y(t, n)}{X(t, n)}\right]
$$

para:

$X(t, n)=I(t, n) \cdot I(t, n+1)+Q(t, n) \cdot Q(t, n+1)$

$$
Y(t, n)=Q(t, n) \cdot I(t, n+1)-I(t, n) \cdot Q(t, n+1)
$$

Portanto, após a aplicação de um demodulador de quadratura aos sinais de ecos ultrassônicos, obtêm-se os componentes em fase e quadratura destes sinais, os quais são utilizados em (7) para se estimar a representação do sinal de vibração do meio $s(t, n)$. Uma vez que $s(t, n)$ é contaminado por ruído, o mesmo é usado como parâmetro de entrada em um filtro de Kalman, para estimar sua amplitude e fase.

\section{Filtro de Kalman}

Para uma vibração com amplitude muito pequena, geralmente de alguns micrômetros, o sinal $s(t, n)$ possui um baixo valor para a relação sinal ruído, o que dificulta a estimativa confiável para sua amplitude e fase (Zheng et al., 2007). Uma maneira encontrada de superar este problema foi o uso do filtro de Kalman em $s(t, n)$ para extrair informações relativas a vibração do meio (Zheng et al., 2003).

O filtro de Kalman é um estimador linear de fácil implementação. Utiliza um modelo de espaço de estados para estimar recursivamente as variáveis de estado, de acordo com o erro quadrático médio mínimo (MMSE). Na hipótese de todas as variáveis aleatórias utilizadas na modelagem serem independentes e com distribuição gaussiana, o filtro de Kalman é mais eficiente que outros estimadores baseados no MMSE, pois ele o minimiza (Brown e Hwang, 1992).

Considerando-se o sinal de eco proveniente de uma estrutura vibrante, então o sinal $s(t, n)$ em (7) é discreto em $n$ e sua estimativa, usando o filtro de Kalman, é representada por:

$$
y_{n}=\beta_{n} \cos \left(\omega_{\mathrm{s}} n T+\varphi_{s, n}\right)+n_{n}
$$

onde o termo $n_{n}$ representa ruído branco. O filtro de Kalman estima valores de $\beta_{n}$ para amplitude e de $\varphi_{\mathrm{s}, \mathrm{n}}$ para a fase, a partir de valores experimentais de $y_{n}$ obtidos como valores discretos de $s(t, n)$, com
$1 \leq n \leq N$, sendo $N$ o número total de pulsos que formam a salva de senoides usadas para excitar o transdutor de ultrassom. A aplicação do filtro leva em conta o conhecimento da frequência de vibração da estrutura que gera o sinal de eco (Zheng et al., 2003).

Utilizando-se o modelo de espaço de estado discreto, a Equação 10 pode ser reescrita como:

$$
\begin{aligned}
y_{n}= & {\left[\cos \left(\omega_{s} n T\right),-\operatorname{sen}\left(\omega_{s} n T\right)\right] . } \\
& {\left[\beta_{n} \cos \left(\phi_{\mathrm{s}, \mathrm{n}}\right), \beta_{n} \operatorname{sen}\left(\phi_{\mathrm{s}, \mathrm{n}}\right)\right]^{\mathrm{T}}+n_{n} }
\end{aligned}
$$

Onde $A^{\mathrm{T}}$ representa a matriz transposta de $A$.

As variáveis de estado são definidas como na equação a seguir:

$\left[\mathrm{x}_{n}\right]=\left[\begin{array}{l}x_{n}(1) \\ x_{n}(2)\end{array}\right]=\left[\begin{array}{l}\beta_{n} \cos \left(\varphi_{s, n}\right) \\ \beta_{n} \operatorname{sen}\left(\varphi_{s, n}\right)\end{array}\right]$

A equação que relaciona a variável de estado atual à variável de estado futuro é dada por (Zheng et al., 2007):

$\left[x_{n+1}\right]=\left[\begin{array}{cc}1 & 0 \\ 0 & 1\end{array}\right] \cdot\left[x_{n}\right]+w_{n}$

onde $w_{n}$ refere-se às amplitudes da sequência de ruídos brancos, que se aproximam de zero quando se tratam dos ruídos inseridos nas medidas da vibração do meio em decorrência do sistema eletrônico. A matriz identidade é denominada matriz de transição (Zheng et al., 2007).

$\mathrm{O}$ valor medido $y_{n}$ está relacionado às variáveis de estado por meio do vetor de medição $H$, de acordo com a equação de medição dada a seguir (Zheng et al., 2003; 2004; 2007):

$y_{n}=H_{n} x_{n}+n_{n}$

Onde $H_{n}=\left[h_{k}(1) h(2)\right]=\left[\cos \left(\omega_{s} n T\right)-\operatorname{sen}\left(\omega_{s} n T\right)\right]$.

De acordo com (14), a amplitude e a fase para $y_{n}$ são obtidas das variáveis de estado através das seguintes equações:

$\beta_{n}=\sqrt{x_{n}^{2}(1)+x_{n}^{2}(2)}$

$\phi_{s, n}=\tan ^{-1}\left[\frac{x_{n}(2)}{x_{n}(1)}\right]$

As etapas para a implementação do filtro de Kalman são descritas a seguir (Brown e Hwang, 1992):

Condição inicial:

$P_{1}^{-}=p_{0} I$

$x_{1}^{-}=x_{0}$

Cálculo do Ganho de Kalman na $n$-ésima etapa: 


$$
G_{n}=P_{n}^{-} H_{n}^{T}\left(H_{n} P_{n}^{-} H_{n}^{T}+R\right)^{-1}
$$

Cálculo da estimativa atualizada da variável de estado:

$\hat{x}_{n}=\hat{x}_{n-1}^{-}+G_{n}\left[y_{n}-H_{n} \hat{x}_{n-1}^{-}\right]$

Cálculo da matriz de covariância do erro:

$P_{n}=\left(I-G_{n} H_{n}\right) P_{n}^{-}$

Estimativas futuras:

$\hat{x}_{n+1}^{-}=\Phi_{n} \hat{x}_{n}$

$P_{n+1}^{-}=\Phi_{n} P_{n} \Phi_{n}^{T}+Q$

onde $I$ é uma matriz identidade $(2 \times 2)$, $R$ é a variância do ruído branco $n_{n}, \Phi_{n}$ é a matriz de transição e $Q$ a matriz de covariância do ruído do processo. O sinal negativo sobrescrito indica uma estimativa a priori, a qual pode ser obtida por meio de uma estimativa futura ou de um valor inicial. Zheng et al. (2007) mencionam que é possível atribuir um valor inicial elevado para $P_{0}$. Além disso, ele é inversamente proporcional à relação sinal ruído. Já o valor inicial da variável de estado $x_{0}$, pode ser considerado nulo nas condições iniciais. $P_{n}$ é uma matriz $2 \times 2$ que representa a covariância do erro das variáveis de estado.

\section{Método experimental}

Foi montado um sistema experimental para testar o DUmV, incluindo uma membrana de poliuretano com vibração imposta por um alto-falante com potência máxima de $3 \mathrm{~W}$, impedância de $1 \Omega$ e resposta em frequência, tipicamente, de $20 \mathrm{~Hz}$ a $18 \mathrm{kHz}$, conforme ilustrado na Figura 1. O sistema de ultrassom operou com o transdutor de detecção não focalizado (distância do campo próximo de 7,6 cm) $T_{\text {det }}$ (Olympus NDT Inc., modelo V326) excitado por uma salva de senoides com frequência de $4,96 \mathrm{MHz}$ e 5 ciclos. A membrana vibrante é parte da parede do reservatório de água e a face de $T_{\operatorname{det}}$ foi posicionada a aproximadamente $7 \mathrm{~cm}$ dela, de maneira que o feixe de ultrassom emitido por $T_{\text {det }}$ incidisse perpendicularmente a ela. O sinal de eco correspondente foi capturado e processado para se determinar a vibração da membrana. $\mathrm{O}$ alto-falante foi excitado pelo gerador de funções (Hewlett-Packard, modelo HP 8116A) configurado para gerar um sinal senoidal com frequência de $150 \mathrm{~Hz}$ e amplitude de $1 \mathrm{~V}$ (pico a pico).

Para excitar o transdutor ultrassônico, cuja frequência central nominal é $4,96 \mathrm{MHz}$, e captar os sinais refletidos da membrana em movimento, usou-se a placa TB 1000 (MATEC Instruments) instalada no barramento ISA de um microcomputador pessoal (Pentium $^{\circledR}$, 64 MB RAM e sistema operacional Microsoft ${ }^{\circledR}$ Windows 95). Esta placa foi utilizada para excitar o transdutor de ultrassom $T_{\text {det }}$ na forma de uma salva de senoides (burst) com frequência de repetição de pulsos de $2 \mathrm{kHz}$, duração do pulso de $1 \mu \mathrm{s}$, ganho de $20 \mathrm{~dB}$, trigger interno e alta tensão de excitação ( $300 \mathrm{~V}$ pico a pico). Além disso, a placa foi configurada para funcionar na forma pulso-eco, sendo com isto capaz de fornecer os sinais de eco capturados por $T_{\text {det }}$, já amplificados e, se necessário, filtrados. Uma saída dessa placa, denominada rec out, possibilitou exibir e capturar o sinal refletido pela membrana em um osciloscópio digital (Tektronix, modelo DPO 3032), operando com uma taxa de amostragem de $125 \mathrm{MA} / \mathrm{s}$ e memória de $5 \mathrm{MB}$, proporcionando uma aquisição correspondente a 40 ms de sinal de RF (radiofrequência) coletado. Os dados armazenados na memória do osciloscópio foram transferidos, via interface USB, para um netbook (Intel ${ }^{\circledR}$ Atom $^{\mathrm{TM}}$, CPU N270, 1,60 GHz, 1 RAM, Microsoft ${ }^{\circledR}$ Windows XP Home Edition), usando um programa de comunicação desenvolvido em LabView ${ }^{\circledR}$ v. 7.1 (National Instruments). Com os dados armazenados foi realizado o processamento de sinal empregando-se as técnicas DUmV e MCC e com o objetivo de confrontar os resultados das mesmas. O processamento de sinal foi realizado em um microcomputador (Pentium ${ }^{\circledR}$ Dual Core, CPU E5300, 2,60 GHz, 2 GB RAM, Microsoft ${ }^{\circledR}$ Windows XP Professional), usando um programa em $\operatorname{MATLAB}^{\circledR}$ (v. R2009a).

$O$ processamento realizado para a técnica DUmV forneceu os componentes de fase e quadratura. $\mathrm{O}$ sinal $s(t, n)$ foi determinado e passado em um filtro de Kalman para a estimativa de sua amplitude $\beta$, de sua fase inicial e/ou de sua frequência.

Uma sequência de sinais de RF típica adquirida pelo osciloscópio, composta por sinais de excitações da salva de senoides, dos ecos correspondentes e de sinais de reverberações entre transdutor e membrana está mostrada na Figura 2. Nesta figura, apenas um vinte avos do sinal coletado, equivalente a $2 \mathrm{~ms}$, está exibido.

Para estimar a vibração do meio, o passo inicial consistiu em segmentar o sinal de RF capturado pelo osciloscópio, onde cada segmento corresponde a um ciclo da salva de senoides (excitação e ecos). Posteriormente, foi realizado o alinhamento de todos os segmentos, de forma a colocá-los iniciando em um mesmo instante de tempo. Para isso, determinou-se, inicialmente, o módulo do sinal de RF e o resultado passou por filtro Butterworth passa-baixa de ordem 2 e frequência de corte equivalente a um terço da frequência central do sinal de eco capturado pelo 


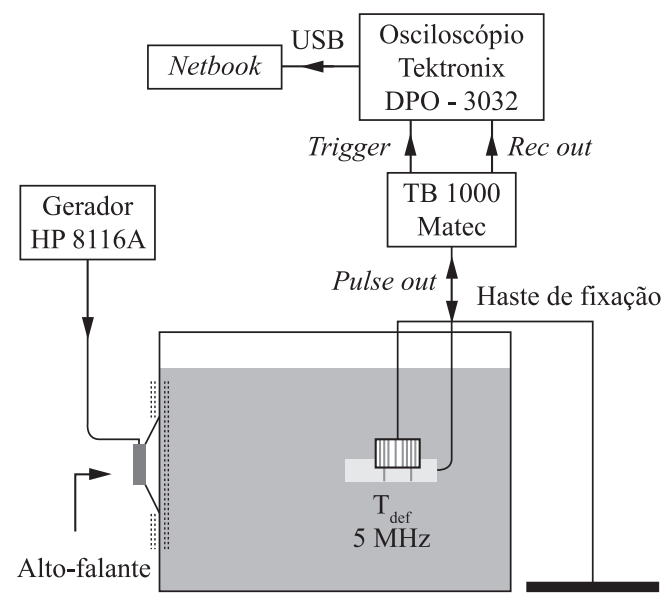

Figura 1. Montagem experimental utilizada para estudar a vibração da membrana. O gerador excita o alto-falante com sinais senoidais de baixa frequência, o que provoca a vibração da membrana. Já a placa TB 1000 é configurada para excitar o transdutor ultrassônico para operar no modo pulso-eco. Os sinais de eco fornecidos na saída rec out da placa são capturados pelo osciloscópio digital e transferidos ao microcomputador através de uma interface USB, onde são processados.

Figure 1. Experimental setup used to study the membrane vibration. The signal generator drives the speaker with a low frequency sinusoidal waveform, forcing the membrane to vibrate. The TB 1000 board was configured to make the ultrasonic transducer working in a pulse-echo mode. The echo signals provided at the board rec out output were captured with a digital oscilloscope and transferred to a microcomputer, by an USB interface, where they were processed.

1/20 do sinal ultrassônico adquirido

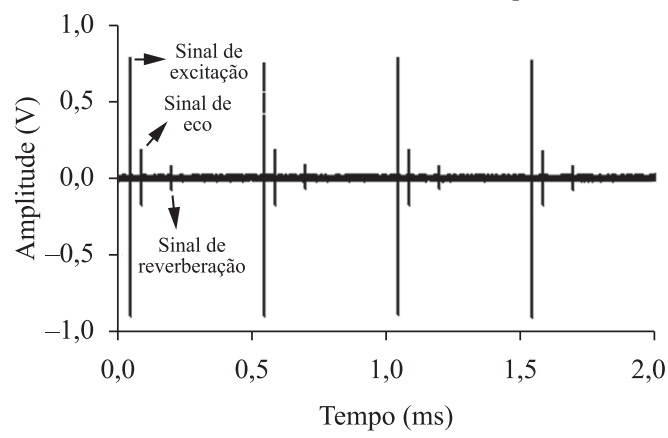

Figura 2. Exemplo de um sinal ultrassônico de RF (radiofrequência) coletado durante $2 \mathrm{~ms}$.

Figure 2. Example of a $R F$ (radiofrequency) ultrasonic signal collected during $2 \mathrm{~ms}$.

transdutor ultrassônico. O sinal filtrado constituiu-se, basicamente, nas envoltórias dos sinais de excitação, de eco e de reverberação. Logo após aplicou-se um offset de $-80 \%$ da amplitude máxima do sinal filtrado e com isso somente parte das envoltórias dos sinais de excitação cruzou o eixo que representa o tempo, ou seja, em $0 \mathrm{~V}$. Do sinal resultante foram encontrados os instantes de cruzamento por zero, no sentido ascendente, das envoltórias relacionadas aos sinais de excitação. A Figura 3 ilustra os processos descritos, porém considerando-se apenas $460 \mu$ s do sinal adquirido para exemplificar o processo.

Uma vez encontrados os pontos ascendentes de cruzamento de zero correspondentes a cada segmento, o instante inicial para cada segmento foi tomado antecedendo 150 pontos amostrais no segmento. Uma vez definido o início do segmento, o seu comprimento foi definido correspondendo a uma quantidade de dados amostrais capaz de conter todo o trecho entre o sinal de excitação e o sinal de eco de interesse. Tipicamente, cada segmento foi constituído de 14.001 pontos, o que corresponde a $112 \mu$ s de duração para uma frequência de amostragem de $125 \mathrm{MHz}$. A Figura 4 exibe parte (cerca de $100 \mu \mathrm{s}$ ) de um segmento típico para todos os experimentos contendo a excitação e o sinal de eco correspondente.

Para alinhar os segmentos foi necessário encontrar um ponto de referencia em cada um deles. Para isso, em cada segmento aplicou-se um offset de $80 \%$ da amplitude máxima do sinal do segmento correspondente e em seguida encontrou-se o primeiro ponto ascendente positivo, o qual foi adotado como o ponto inicial do respectivo segmento. Esse procedimento foi repetido para os demais segmentos e cada um deles passou a ter seu ponto inicial. A Figura 5 apresenta parte de três segmentos consecutivos, de um mesmo experimento, alinhados.

A quantidade de segmentos do sinal é obtida levando-se em consideração o tamanho de memória (5 MB) do osciloscópio, a frequência de amostragem (125 MHz) e o período de repetição dos pulsos (PRP) da salva de senoides. Sendo assim, com o valor de PRP empregado neste trabalho $(0,5)$ obtiveram-se 80 segmentos.

Após a segmentação do sinal de RF e alinhamento dos segmentos foram gerados sinais seno e cosseno, sincronizados com a excitação, para serem usados na determinação dos componentes de fase e quadratura dos sinais de eco. Esses sinais senoidais foram gerados com amplitude de $1 \mathrm{~V}$ pico a pico e na frequência de 4,96 MHz. Para a sincronização dos mesmos com a excitação foi necessário encontrar a fase inicial desses sinais senoidais. Para isto, foram detectados os instantes de cruzamento por zero do sinal de excitação contido em todos os segmentos. Esses instantes de cruzamento por zero foram ordenados em ordem crescente e para o primeiro deles foi determinado se o mesmo era ascendente ou descendente. Caso ascendente, a fase total do cosseno corresponderia a $3 \pi / 2$ radianos e em caso contrário corresponderia a $5 \pi / 2$ radianos. Para os cruzamentos subsequentes (tipicamente 4 no mesmo segmento), a fase total do cosseno corresponderia a múltiplos de $3 \pi / 2$ ou 


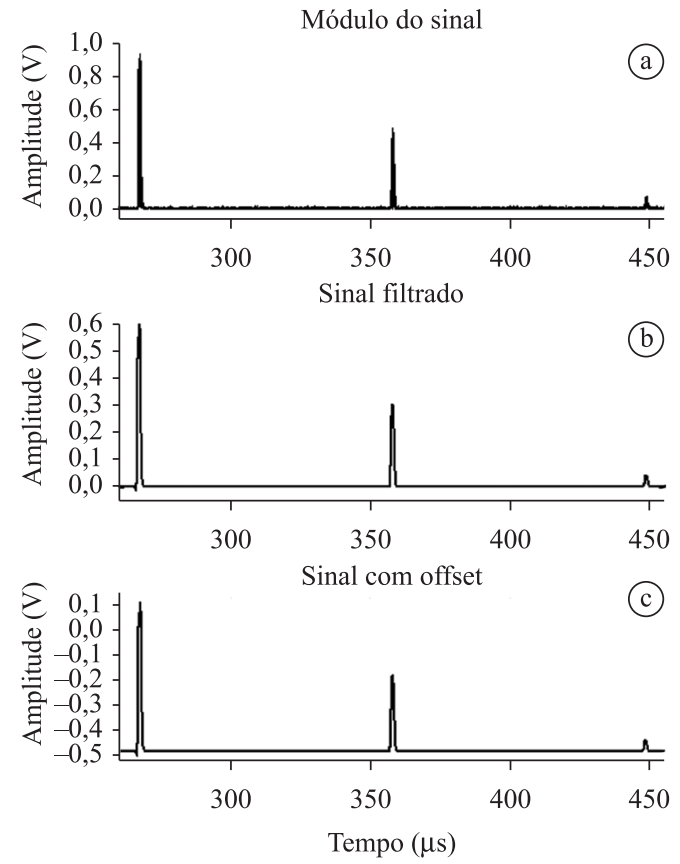

Figura 3. Processo empregado para encontrar o ponto ascendente do sinal de excitação, por cruzamento pelo zero. a) Módulo do sinal de RF após tirar o nível DC; b) Envoltórias dos sinais de excitação, eco e reverberação, obtidas por filtragem do módulo do sinal de RF; c) Sinal filtrado após a aplicação de um offset de $-80 \%$ da amplitude máxima do sinal de RF, sendo que somente o envelope do sinal de excitação cruza o nível de zero volt.

Figure 3. Zero-crossing method used to find the ascending point of the excitation signal. a) RF signal magnitude after the DC level being removed; b) Excitation, echo and reverberation signal envelopes obtained from the filtered RF signal magnitude; c) Filtered signal shifted down byan offset of $-80 \%$ with respect to the $R F$ signal maximum amplitude. Only the excitation signal envelope crosses zero voltage level.

de $5 \pi / 2$, caso fossem ascendentes ou descendentes, respectivamente. A fase inicial considerada para os sinais senoidais foi a média dos valores correspondentes encontrados para todos os cruzamentos por zero do sinal de excitação. Sendo assim, foi encontrada a média das fases de cada segmento. A moda desses valores foi usada como a fase dos sinais senoidais.

A Figura 6 mostra que método desenvolvido funcionou adequadamente, pois o sinal de cosseno gerado se encaixou muito bem no sinal de excitação.

Uma vez gerados os sinais de cosseno e seno, eles foram multiplicados, separadamente, por todos os segmentos. Os dois produtos resultantes passaram, cada um, por um filtro passa-baixas do tipo Butterworth com ordem 2 e frequência de corte igual a um terço de 4,96 MHz. As saídas dos filtros continham os componentes em fase e em quadratura dos sinais de eco. Foram tomados os valores dos componentes em fase ou em quadratura correspondentes ao instante central do sinal de eco, com os quais se determinou o

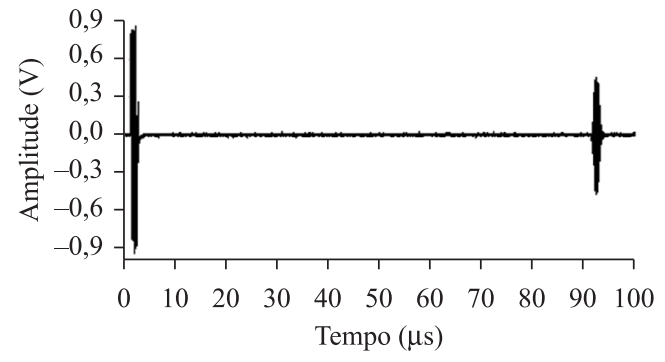

Figura 4. Parte de um segmento típico do sinal adquirido, contendo a excitação e um sinal de eco.

Figure 4. Part of a typical segment of the collected signal, containing the excitation and echo signals.

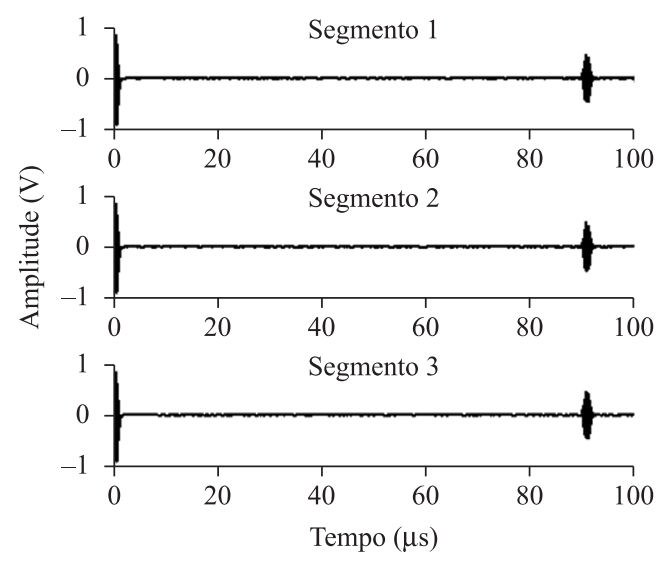

Figura 5. Parte de três segmentos consecutivos alinhados, os quais possuem duração de 100 microssegundos.

Figure 5. Part of three consecutive aligned segments, each one with 100 microseconds of duration.

valor de $s(t, n)$, para $1 \leq n \leq N$, sendo $N$ a quantidade de segmentos.

A Figura 7 mostra um exemplo do sinal $s(t, n)$ calculado utilizando o método descrito anteriormente e o sinal de vibração da membrana estimado pelo filtro de Kalman.

Neste trabalho não se implementou um sincronismo entre a excitação do alto-falante e a do $T_{\text {det }}$. Com isto, não se reproduziram os valores da fase de $s(t, n)$ correspondentes a experimentos repetidos.

\section{Resultados}

A Figura 8 mostra o resultado da utilização do filtro de Kalman para encontrar a frequência de vibração da membrana. Para isso, mantendo-se o alto-falante excitado por um sinal com amplitude de $6 \mathrm{~V}$ pico a pico e frequência de $150 \mathrm{~Hz}$, o filtro de Kalman foi empregado repetidas vezes com o valor da frequência, utilizada na rotina do filtro, variando entre 10 e $750 \mathrm{~Hz}$ 
e com passo de $1 \mathrm{~Hz}$. Para cada valor de frequência tomou-se a amplitude do sinal de vibração estimado pelo filtro e a frequência de vibração estimada pelo filtro foi considerada como sendo aquela com a qual se obteve o maior valor de amplitude do sinal de vibração estimado. Neste caso, a frequência estimada foi de $149 \mathrm{~Hz}$

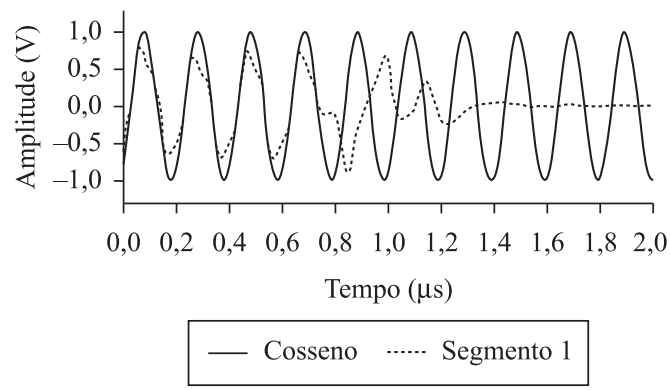

Figura 6. Detalhes do sinal cosseno e do primeiro segmento, o qual está representado pelo sinal de excitação. Nota-se que eles se encaixam bem.

Figure 6. Details of the cosine and first segment, which is represented by the excitation signal. Note that they match well.

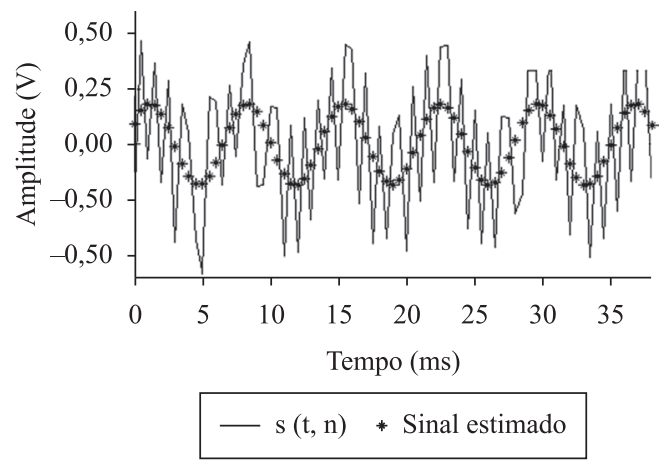

Figura 7. Exemplo do sinal de vibração da membrana detectado e o sinal correspondente estimado pelo filtro de Kalman.

Figure 7. Example of the detected membrane vibration signal and the corresponding signal estimated by the Kalman filter.

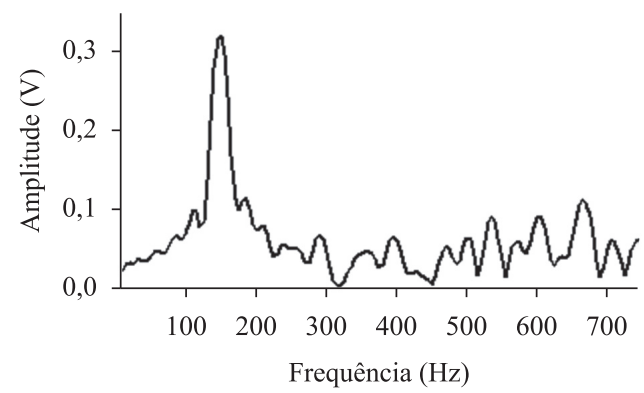

Figura 8. Amplitude de vibração em função frequência, usada no cálculo do filtro de Kalman, variando de 10 a $750 \mathrm{~Hz}$, com passos de $1 \mathrm{~Hz}$

Figure 8. Vibration amplitude as a function of the frequency, used to calculate the Kalman filter, spanning from 10 to $750 \mathrm{~Hz}$ in steps of $1 \mathrm{~Hz}$.
Outra etapa desde experimento consistiu na utilização do filtro de Kalman para detectar a frequência de vibração da membrana, quando o alto-falante foi excitado com um sinal senoidal de $12 \mathrm{~V}$ pico a pico. Para as frequências usadas no gerador com valores de $100,130,180,200$ e $280 \mathrm{~Hz}$, as correspondentes frequências estimadas pelo filtro foram $99,128,178$, 197 e $272 \mathrm{~Hz}$, respectivamente.

Conhecida a frequência de vibração da membrana utilizou-se o filtro de Kalman para estimar o sinal de vibração. A Figura 9 ilustra a forma de onda estimada para o sinal de vibração correspondente ao $s(t, n)$ obtido com o gerador configurado para produzir um sinal senoidal de $150 \mathrm{~Hz}$ e $6 \mathrm{~V}$ pico a pico.

$\mathrm{O}$ passo seguinte consistiu em determinar a amplitude mínima da forma de onda do sinal de vibração da membrana que o $\mathrm{DUmV}$ conseguiria estimar. Para isso o sinal senoidal de excitação do alto-falante foi mantido na frequência de $150 \mathrm{~Hz}$ e foram considerados os valores de $0,5,1,3,6,9$, 12 e $15 \mathrm{~V}$ pico a pico. Para a excitação de $0,5 \mathrm{~V}$ pico a pico, o filtro de Kalman do DUmV não convergiu para uma estimativa da forma de onda do sinal de vibração da membrana. Para os demais valores, os resultados da média ( \pm 1 desvio padrão) da amplitude da forma de onda do sinal de vibração da membrana, estimado pelo DUmV são apresentados na Tabela 1. Para efeitos práticos, considera-se como $1,33 \mu \mathrm{m}$ a amplitude mínima de vibração que o DUmV foi capaz de estimar.

\section{Discussão}

A varredura realizada pelo filtro de Kalman, para estimar a frequência de vibração da membrana, conseguiu extrair valores de frequências correspondentes às dos sinais de excitação da membrana, o que garante que a vibração estimada estava relacionada com a frequência de vibração fundamental da membrana. $\mathrm{O}$ erro máximo das medidas foi menor que $3 \%$.

Como era esperado, à medida que a tensão no gerador foi aumentada, a amplitude da vibração estimada pelo filtro de Kalman também teve uma elevação em seus valores. O DUmV conseguiu extrair a vibração do meio para várias frequências de vibração $(100,130,150,200,280 \mathrm{~Hz})$, mantendo-se constante o nível de excitação do alto-falante. Nesse caso, a amplitude de vibração e a frequência variaram em sentidos opostos. Os resultados obtidos para este tipo de vibração apresentam o mesmo efeito observado no estudo de Zheng et al. (2007) para a vibração do meio gerada pela propagação da onda de cisalhamento, pois eles obtiveram valores de amplitude de vibração menores à medida que a frequência da 


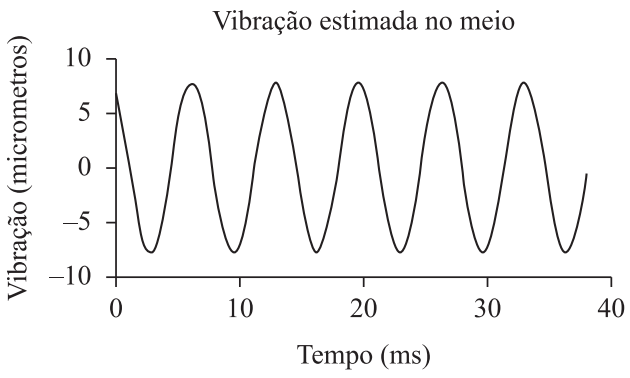

Figura 9. Vibração estimada a partir da representação do sinal de vibração extraída do meio, quando o gerador excitou o alto-falante com um sinal senoidal com frequência de $150 \mathrm{~Hz}$ e amplitude de $6 \mathrm{~V}$ pico a pico.

Figure 9. Estimated membrane vibration with the speaker driven by a sinusoidal waveform with a 6 V peak-to-peak amplitude and at $150 \mathrm{~Hz}$.

onda de cisalhamento aumentou de $100 \mathrm{~Hz}$ para $500 \mathrm{~Hz}$ (passo de $100 \mathrm{~Hz}$ ).

Para apurar o método desenvolvido quanto à confiabilidade, nos valores obtidos para a amplitude da forma de onda do sinal de vibração da membrana estimado pelo filtro de Kalman, foi empregada uma nova técnica para processar os sinais já coletados, na qual buscou-se determinar a distância entre a face do transdutor $\left(\mathrm{T}_{\text {det }}\right)$ e a membrana, ao longo do tempo. Para isto foi medido o atraso entre o sinal de excitação e o sinal de eco, usando-se o método de correlação cruzada (MCC) entre os mesmos. A diferença entre os atrasos medidos foi de 35,36 ns para um sinal de excitação empregado no alto-falante com amplitude de $9 \mathrm{~V}_{\mathrm{pp}}$ e $150 \mathrm{~Hz}$. Após subtrair da forma de onda da distância o seu valor médio, a forma de onda do sinal de vibração da membrana foi obtida. Os resultados obtidos para a amplitude de vibração da membrana, utilizando o MCC, são apresentados na Tabela 1. Na Figura 10 pode ser observado o $s(t, n)$ estimado empregando-se o MCC, que é obtido com o gerador configurado para produzir um sinal senoidal de $150 \mathrm{~Hz}$ e $6 \mathrm{~V}$ pico a pico.

Os resultados obtidos pelos métodos DUmV e MCC apresentados na Tabela 1 foram confrontados com a utilização do teste $t$ de Student, o qual demonstrou não haver diferença significativa entre as médias das amplitudes de vibração da membrana, quando o nível de significância foi de 0,01 . Isso indica que o DUmV foi implementado de forma confiável para estimar a vibração da membrana. Considerando-se a praticidade entre os métodos DUmV e MCC, o primeiro tem a vantagem de ser empregado para sinais de baixa frequência (componentes em fase e em quadratura), enquanto o segundo opera diretamente com os sinais de eco (RF). Embora no presente estudo a geração dos componentes em fase e quadratura tenha sido realizada através do processamento de sinais digitais,
Tabela 1. Amplitudes (média \pm desvio padrão) estimadas da vibração da membrana, usando-se o DUmV e o Método de Correlação Cruzada (MCC) em função da amplitude pico-pico $\left(\mathrm{V}_{\mathrm{pp}}\right)$ do sinal de excitação do alto-falante em $150 \mathrm{~Hz}$.

Table 1. Membrane vibration estimated amplitude (mean \pm standard deviation), using the UDmV and Cross-Correlation Method (CCM) as a function of the peak-to-peak amplitude $\left(V_{p p}\right)$ of the speaker the excitation signal at $150 \mathrm{~Hz}$.

\begin{tabular}{ccc}
\hline $\begin{array}{c}\mathbf{V}_{\mathbf{p p}} \\
{[\mathbf{V}]}\end{array}$ & $\begin{array}{c}\text { Amplitude } \\
(\mathbf{D U m V}) \\
{[\boldsymbol{\mu m}]}\end{array}$ & $\begin{array}{c}\text { Amplitude } \\
(\mathbf{M C C}) \\
{[\boldsymbol{\mu m}]}\end{array}$ \\
\hline 1 & $1,33 \pm 0,30$ & $1,30 \pm 0,21$ \\
3 & $3,80 \pm 0,23$ & $3,85 \pm 0,27$ \\
6 & $7,68 \pm 0,32$ & $7,92 \pm 0,20$ \\
9 & $11,74 \pm 0,50$ & $12,12 \pm 0,44$ \\
12 & $15,57 \pm 0,50$ & $16,03 \pm 0,44$ \\
15 & $20,24 \pm 0,28$ & $20,55 \pm 0,33$ \\
\hline
\end{tabular}

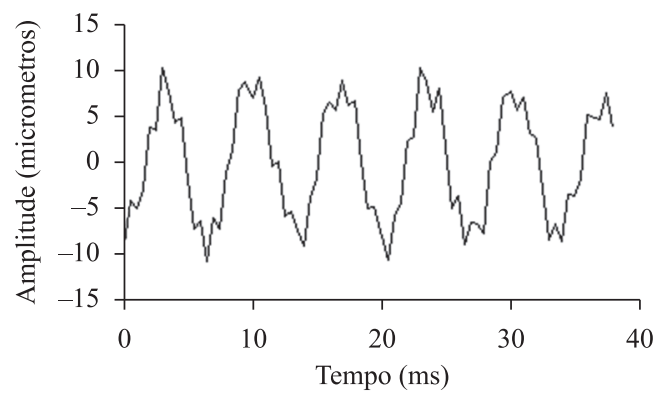

Figura 10. Exemplo do sinal de vibração da membrana s(t,n) detectado usando-se o MCC, quando o gerador excitou o alto-falante com um sinal senoidal com frequência de $150 \mathrm{~Hz}$ e amplitude de $6 \mathrm{~V}$ pico a pico. Figure 10. Example of the detected membrane vibration signal $s(t, n)$ using CCM, with the speaker driven by a sinusoidal waveform with a 6 V peak-to-peak signal amplitude and at $150 \mathrm{~Hz}$.

é possível gerar, em tempo real, os componentes em fase e quadratura usando hardware específico e de baixa complexidade. Portanto, com o método DUmV é possível utilizar sistemas de aquisição de sinais com frequência de amostragem na faixa de $\mathrm{kHz}$, ao passo que com o MCC torna-se necessário o uso de sistemas de aquisição com frequência de amostragem na faixa de MHz. Daí a praticidade do DUmV sobre o MCC, uma vez que custos menores e melhor precisão (temporal e no número de bits) de digitalização de sinais podem ser conseguidos com sistemas de aquisição para baixas frequências.

Diante dos resultados apresentados neste trabalho, observa-se que o DUmV foi capaz de detectar microvibrações da membrana de poliuretano, com algumas amplitudes da mesma ordem de grandeza que os valores encontrados no meio biológico (micrometros) segundo as metodologias usadas por Bercoff et al. (2004), Zheng et al. (2007) e Chen et al. (2009). 


\section{Conclusão}

Com os resultados obtidos neste trabalho, conclui-se que o DUmV foi capaz de detectar microvibrações do meio, as quais são da mesma ordem de grandeza que os valores encontrados na literatura. Com isso é possível partir para uma etapa seguinte, na qual o DUmV será aplicado à detecção de vibração no meio devido à propagação de ondas de cisalhamento.

\section{Agradecimentos}

Os autores agradecem ao $\mathrm{CNPq}$ pela bolsa de estudos e pelo suporte financeiro para a realização dos experimentos.

\section{Referências}

ANDERSON, W. A. D. Pathology. 8th ed. Saint Louis: CW Mosby Co., 1985. 2074 p.

BENECH, N. Elastografia Ultrasonora de Medios Viscoelásticos con ondas de Cizalla de baja Frecuencia. 2004. $121 \mathrm{f}$. Disertación (Mestrado Desarrollo de las Ciencias Básicas)Universidad de la Republica, Montevideo, 2004.

BERCOFF, J.; MULLER, M.; TANTER, M.; FINK, M. Study of viscous and elastic properties of soft tissues using supersonic shear imaging. In: IEEE INTERNATIONAL ULTRASONICS SYMPOSIUM, 2003, Honolulu, Hawaii. Proceedings... IEEE, 2003. p. 925-928. http://dx.doi. org/10.1109/TUFFC.2004.1295425

BERCOFF, J.; TANTER, M.; FINK, M. Supersonic shear imaging: A new technique for soft tissue elasticity mapping. IEEE Transactions on Ultrasonics Ferroelectrics and Frequency Control, v. 51, n. 4, p. 396-409, 2004.

BROWN, R. G.; HWANG, P. Y. C. Introduction to Random Signals and Applied Kalman Filtering. 2th ed. New York: John Wiley and Sons, 1992. $512 \mathrm{p}$.

CARSTENSEN, E. L.; PARKER, K. J.; LERNER, R. M. Elastography in the management of liver disease. Ultrasound in Medicine and Biology, v. 34, n. 10, p. 1535-1546, 2008. http://dx.doi.org/10.1016/j.ultrasmedbio.2008.03.002

CASTERA, L.; FORNS, X.; ALBERTI, A. Non-invasive evaluation of liver fibrosis using transient elastography. Journal of Hepatology, v. 48, n. 5, p. 835-847, 2008. PMid:18334275. http://dx.doi.org/10.1016/j.jhep.2008.02.008

CATHELINE, S.; GENNISSON, J. L.; DELON, G.; FINK, M.; SINKUS, R.; ABOUELKARAM, S.; CULIOLI, J. Measuring of viscoelastic properties of homogeneous soft solid using transient elastography: an inverse problem approach. Journal of the Acoustical Society of America, v. 116 , n. 6 , p. 3734-3741, 2004. PMid:15658723. http://dx.doi.org/10.1121/1.1815075

CATHELINE, S.; SANDRIN, L.; GENNISSON, J. L.; TANTER, M.; FINK, M. Ultrasound-based noninvasive shear elasticity probe for soft tissues. In: IEEE INTERNATIONAL ULTRASONICS SYMPOSIUM, 2000, San Juan, Puerto Rico. Proceedings... IEEE, 2000. p. 1799-1801.

CATHELINE, S.; THOMAS, J. L.; WU, F.; FINK, M. Diffraction field of a low frequency vibrator in soft tissues using transient elastography. IEEE Transactions on
Ultrasonics, Ferroelectrics and Frequency Control, v. 46, n. 4, p. 1013-1019, 1999a. PMid:18238506. http://dx.doi.org/10.1109/58.775668

CATHELINE, S.; WU, F.; FINK, M. A solution to diffraction biases in sonoelasticity: the acoustic impulse technique. Journal of the Acoustical Society of America, v. 105, n. 5, p. 2941-2950, 1999b. PMid:10335643. http://dx.doi. org/10.1121/1.426907

CHEN, S.; URBAN, M. W.; PISLARU, C.; KINNICK, R.; ZHENG, Y.; YAO, A.; GREENLEAF, J. F. Shearwave dispersion ultrasound vibrometry (SDUV) for measuring tissue elasticity and viscosity. IEEE Transactions on Ultrasonics, Ferroelectrics and Frequency Control, v. 56, n. 1, p. 55-62, 2009. PMid:19213632. PMCid:2658640. http://dx.doi.org/10.1109/TUFFC.2009.1005

COCHLIN, D. L.; GANATRA, R. H.; GRIFFITHS, D. F. Elastography in the detection of prostatic cancer. Clinical Radiology, v. 57, n. 11, p. 1014-1020, 2002. PMid:12409113. http://dx.doi.org/10.1053/crad.2002.0989

DE LÉDINGHEN, V.; VERGNIOL, J. Transient elastography (FibroScan). Gastroenterologie Clinique et Biologique, v. 32 , n. 6 , p. $58-67,2008$. Suplemento 1.

DEFFIEUX, T.; MONTALDO, G.; TANTER, M.; FINK, M. Shear wave spectroscopy for in vivo quantification of human soft tissues visco-elasticity. IEEE Transactions on Medical Imaging, v. 28, n. 3, p. 313-322, 2009. PMid:19244004. http://dx.doi.org/10.1109/TMI.2008.925077

GAO, L.; ALAM, S. K.; PARKER, K. J. New vibration theory for sonoelasticity imaging. In: IEEE INTERNATIONAL ULTRASONICS SYMPOSIUM, 1993, Baltimore. Proceedings... IEEE, 1993 p. 879-882.

GAO, L.; PARKER, K. J.; ALAM, S. K.; LERNER, R. M. Sonoelasticity imaging: theory and experimental verification. Journal of the Acoustical Society of America, v. 97, n. 6, p. 3875-3886, 1995. PMid:7790663. http://dx.doi. org/10.1121/1.412399

GARRA, B. S.; CESPEDES, E. I.; OPHIR, J.; SPRATT, S. R.; ZUURBIER, R. A.; MAGNANT, C. M.; PENNANEN, M F. Elastography of breast lesions: initial clinical results. Radiology, v. 202, n. 1, p. 79-86, 1997.

GENNISSON, J. L.; CLOUTIER, G. Sol-gel transition in agar-gelatin mixtures studied with transient elastography. IEEE Transactions on Ultrasonics, Ferroelectrics and Frequency Control, v. 53, n. 4, p. 716-723, 2006. http://dx.doi.org/10.1109/TUFFC.2006.1621498

GENNISSON, J. L.; LEROUGE, S.; CLOUTIER, G. Assessment by transient elastography of the viscoelastic properties of blood during clotting. Ultrasound in Medicine and Biology, v. 32, n. 10, p. 1529-1537, 2006. http://dx.doi. org/10.1016/j.ultrasmedbio.2006.06.008

GENNISSON, J. L.; YU, F.; CLOUTIER, G. Analysis of blood clot formation with transient elastography: Similarity with sol-gel transition in Agar-gelatin phantoms. In: IEEE INTERNATIONAL ULTRASONICS, FERROELECTRICS, AND FREQUENCY CONTROL; ANNIVERSARY JOINT CONFERENCE, 50., 2004, Montreal. Proceedings... IEEE, 2004. p. 1134-1137.

GIANNOULA, A.; COBBOLD, R. S. Propagation of narrowband shear waves induced by a finite-amplitude radiation force. In: IEEE INTERNATIONAL ULTRASONICS SYMPOSIUM, 2007, New York. Proceedings... IEEE, 2007. p. 2267-2270

GIANNOULA, A.; COBBOLD, R. S. Narrowband shear wave generation by a Finite-Amplitude radiation force: 
The fundamental component. IEEE Transactions on Ultrasonics, Ferroelectrics and Frequency Control, v. 55, n. 2, p. 343-358, 2008. PMid:18334341. http://dx.doi. org/10.1109/TUFFC.2008.653

GIANNOULA, A.; COBBOLD, R. S. Propagation of shear waves generated by a modulated finite amplitude radiation force in a viscoelastic medium. IEEE Transactions on Ultrasonics, Ferroelectrics and Frequency Control, v. 56, n. 3, p. 575-588, 2009. PMid:19411216. http://dx.doi org/10.1109/TUFFC.2009.1074

GREENLEAF, J. F.; FATEMI, M.; INSANA, M. Selected methods for imaging elastic properties of biological tissues. Annual Review of Biomedical Engineering, v. 5, p. 57-78, 2003. PMid:12704084. http://dx.doi.org/10.1146/annurev. bioeng.5.040202.121623

KAMOI, K.; OKIHARA, K.; OCHIAI, A.; UKIMURA, O.; MIZUTANI, Y.; KAWAUCHI, A.; MIKI, T. The utility of transrectal real-time elastography in the diagnosis of prostate cancer. Ultrasound in Medicine and Biology, v. 34, n. 7, p. 1025-1032, 2008. http://dx.doi.org/10.1016/j. ultrasmedbio.2007.12.002

KRUSE, S. A.; SMITH, J. A.; LAWRENCE, A. J.; DRESNER, M. A.; MANDUCA, A.; GREENLEAF, J. F.; EHMAN, R. L. Tissue characterization using magnetic resonance elastography: preliminary results. Physics in Medicine and Biology, v. 45, n. 6, p. 1579-1590, 2000. PMid:10870712. http://dx.doi.org/10.1088/0031-9155/45/6/313

MELODELIMA, D.; BAMBER, J. C.; DUCK, F. A.; SHIPLEY, J. A. Elastography for breast cancer diagnosis using radiation force: system development and performance evaluation. Ultrasound in Medicine and Biology, v. 32, n. 3, p. 387-396, 2006. http://dx.doi.org/10.1016/j.ultrasmedbio.2005.12.003

MELODELIMA, D.; BAMBER, J. C.; DUCK, F. A.; SHIPLEY, J. A.; XU, L. Transient elastography using impulsive ultrasound radiation force: a preliminary comparison with surface palpation elastography. Ultrasound in Medicine and Biology, v. 33, n. 6, p. 959-969, 2007. http://dx.doi. org/10.1016/j.ultrasmedbio.2006.12.004

NIGHTINGALE, K.; McALEAVEY, S.; TRAHEY, G. Shearwave generation using acoustic radiation force: in vivo and ex vivo results. Ultrasound in Medicine and Biology, v. 29 , n. 12 , p. $1715-1723,2003$. http://dx.doi.org/10.1016/j. ultrasmedbio.2003.08.008

NIGHTINGALE, K.; PALMERI, M. L.; NIGHTINGALE, R. W.; TRAHEY, G. E. On the feasibility of remote palpation using acoustic radiation force. Journal of the Acoustical Society of America, v. 110, n. 1, p. 625-634, 2001. PMid:11508987. http://dx.doi.org/10.1121/1.1378344

NIGHTINGALE, K.; SOO, M. S.; NIGHTINGALE, R.; TRAHEY, G. Acoustic radiation force impulse imaging: in vivo demonstration of clinical feasibility. Ultrasound in Medicine and Biology, v. 28, n. 2, p. 227-235, 2002. http://dx.doi.org/10.1016/S0301-5629(01)00499-9

NITTA, N.; SHIINA, T.; UENO, E. Quantitative assessment and imaging of viscoelastic properties of soft tissue. In: IEEE INTERNATIONAL ULTRASONICS SYMPOSIUM, 2002, Munich. Proceedings... IEEE, 2002. p. 1885-1889.
RENGARAJU, V.; SILVA, A. F. F. D.; SACK, I.; KARGEL, C. A basic study of ultrasonic shear wave elastography in tissue-mimicking phantoms. In: IEEE INTERNATIONAL WORKSHOP ON MEDICAL MEASUREMENTS AND APPLICATIONS, 2009 Cetraro, Italy. Proceedings... IEEE, 2009. p. 55-59.

SAFTOIU, A.; VILMAN, P. Endoscopic ultrasound elastography - a new imaging technique for the visualization of tissue elasticity distribution. Journal of Gastrointestinal and Liver Diseases, v. 15, n. 2, p. 161-165, 2006.

SARVAZYAN, A. P.; RUDENKO, O. V.; SWANSON, S. D.; FOWLKES, J. B.; EMELIANOV, S. Y. Shear wave elasticity imaging: a new ultrasonic technology of medical diagnostics. Ultrasound in Medicine and Biology, v. 24, n. 9, p. 1419-1435, 1998. http://dx.doi.org/10.1016/S03015629(98)00110-0

SPOREA, I.; SIRLI, R.; DELEANU, A.; TUDORA, A.; CURESCO, M.; CORNIANU, M.; LAZAR, D. Comparison of the liver stiffness measurement by transient elastography with the liver biopsy. World Journal of Gastroenterology, v. 14, n. 42, p. 6513-6517, 2008. PMid:19030204. PMCid:2773338. http://dx.doi.org/10.3748/wjg.14.6513

URBAN, M. W.; CHEN, S.; GREENLEAF, J. F. Error in estimates of tissue material properties from shear wave dispersion ultrasound vibrometry. IEEE Transactions on Ultrasonics, Ferroelectrics and Frequency Control, v. 56, n. 4, p. 748-758, 2009. PMid:19406703. PMCid:2756029. http://dx.doi.org/10.1109/TUFFC.2009.1097

YAMAKOSHI, Y.; SATO, J.; SATO, T. Ultrasonic imaging of internal vibration of soft tissue under forced vibration. IEEE Transactions on Ultrasonics, Ferroelectrics and Frequency Control, v. 37, n. 2, p. 45-53, 1990. PMid:18285015. http://dx.doi.org/10.1109/58.46969

ZHENG, Y.; CHEN, S.; TAN, W.; GREENLEAF, J. F. Kalman filter motion detection for vibro-acoustography using pulse echo ultrasound. In: IEEE INTERNATIONAL ULTRASONICS SYMPOSIUM, 2003, Honolulu, Hawaii. Proceedings... IEEE, 2003. p. 1812-1815.

ZHENG, Y.; CHEN, S.; TAN, W.; KINNICK, R.; GREENLEAF, J. F. Detection of tissue harmonic motion induced by ultrasonic radiation force using pulse-echo ultrasound and Kalman filter. IEEE Transactions on Ultrasonics, Ferroelectrics and Frequency Control, v. 54, n. 2, p. 290-300, 2007. http://dx.doi.org/10.1109/TUFFC.2007.243

ZHENG, Y.; CHEN, S.; ZHANG, X.; GREENLEAF, J. Detection of shear wave propagation in an artery using pulse echo ultrasound and Kalman filtering. In: IEEE INTERNATIONAL ULTRASONICS, FERROELECTRICS, AND FREQUENCY CONTROL; ANNIVERSARY JOINT CONFERENCE, 50., 2004, Montreal. Proceedings... IEEE, 2004. p. 1251-1253.

ZHU, Q. L.; JIANG, Y. X.; LIU, J. B.; SUN, Q.; DAI, Q.; CHEN, X. Real-Time Ultrasound Elastography: Its Potential Role in Assessment of Breast Lesions. Ultrasound in Medicine and Biology, v. 34, n. 8, p. 1232-1238, 2008. http://dx.doi.org/10.1016/j.ultrasmedbio.2008.01.004

\section{Autores}

